\title{
Article
}

\section{Forces Acting on Objects in Nanopores with Irregularities - Irregular Nanopores as Shape Sensors}

\author{
Mohammad Tajparast ${ }^{1}$ and Mladen I. Glavinović ${ }^{2, *}$ \\ Departments of Civil Engineering and Applied Mechanics ${ }^{1}$ and Physiology ${ }^{2}$, McGill University, Montreal, PQ \\ H3G 1Y6, Canada \\ *Correspondence: Tel.: (514)-398-6002; mladen.glavinovic@mcgill.ca
}

\begin{abstract}
Nanopores with irregularities are promising tools for distinguishing nano-size objects by their shape, but the forces on the object that critically influence its axial and rotational movement are unclear. The physics of the situation was described using the Poisson-Nernst-Planck and NavierStokes equations. With uniformly charged object the axial Coulomb and dielectric pressure (which opposes it and is surprisingly important), control the object's axial movement and rotation. Even without external pressure the hydrodynamic pressure is significant (negative at its upper and positive at its lower surface), but its total value is almost zero. If the object is charged only on the upper surface the axial upper Coulomb pressure is near zero close to the center, but negative near its end (the pressure is zero at the lower surface). The total axial dielectric pressure, which is largely dominated by the pressure at the upper surface, is positive along the length of the object becoming pronounced near its end. The axial hydrodynamic pressure is negative and significant at the upper surface (zero at the lower surface), diminishes in value near the object's end, critically influencing its axial movement, which becomes much faster. At its end the axial dielectric pressure prevails, and controls its rotation.
\end{abstract}

Keywords: Irregular nanopore; Poisson-Nernst-Planck; Navier-Stokes; Maxwell stress tensor

\section{Introduction}

Over the last several decades there has been an explosive growth of nanopore applications in a variety of fields - biomedical, clinical and biochemical (drug delivery, biosensing, protein filtration, dialysis and pathogen isolation), but also in engineering (nanofluidic ionic diodes and transistors, and ion filters). In this study we focused on a nanopore as a molecular bio-sensor. Initially bio-sensing of molecular size worked on the size-exclusion principle [1]. If a pore diameter is comparable to the molecular size, its passage through the pore gives rise to a discrete and transient current blockade that reveals its size. More advanced detection methods build on the notion that transport depends on the pore wall charges [2], but the passage of a bio-molecule or generally a particle is in both cases associated with the conductance change [1].

However, if long nanopores have irregularities nano-size (and meso-size) spherical objects can be distinguished from non-spherical. It is thus possible to evaluate not only volumes and charges of objects, but also their shapes. A nanopore is considered as irregular if its radius is not constant, but changes in a periodic manner. Within irregular nanopores the objects do not move only axially (translational movement), but also rotate. If their shape is spherical, the rotational movement does not lead to current modulation, but if their shape is non-spherical it does. This method is useful in medicine for virus characterization [3]. What is less clear is to what extent various forces contribute to the movement (translational and rotational) of non-spherical objects and this requires simulations.

We first evaluate the electrostatic variables (potential, axial and radial electric fields) and hydrodynamic variables (pressure, axial and radial water velocity) in an irregular nanopore with a 
charged disk, and then calculate the pressures (and forces) on the disk which would control how (and how quickly) it would move axially, and how it would rotate. The transport of $\mathrm{K}^{+}$, glutamate- (or $\left.\mathrm{Glu}^{-}\right), \mathrm{Na}^{+}$and $\mathrm{Cl}^{-}$within a nanopore was simulated using the Poisson-Nernst-Planck equations [4], and was coupled to the transport of water using the Navier-Stokes equations [5]. Several forces acting on the object were considered and calculated at different locations in the nanopore: a) Coulomb force (due to fixed charges on the surface of the object when present in an electric field), b) dielectric force, and c) hydrodynamic pressure. The dielectric force is induced if the electric field is spatially uneven, and if there is a permittivity mismatch at the interface of two dielectrics (in this case between the object and the solution). This induces charges at the object-solution interface, which are not fixed, but nevertheless influence the electrostatics and generates pressures on the object.

\section{Methods}

2.1 Geometry of simulation domain, parameters, constants and boundary conditions

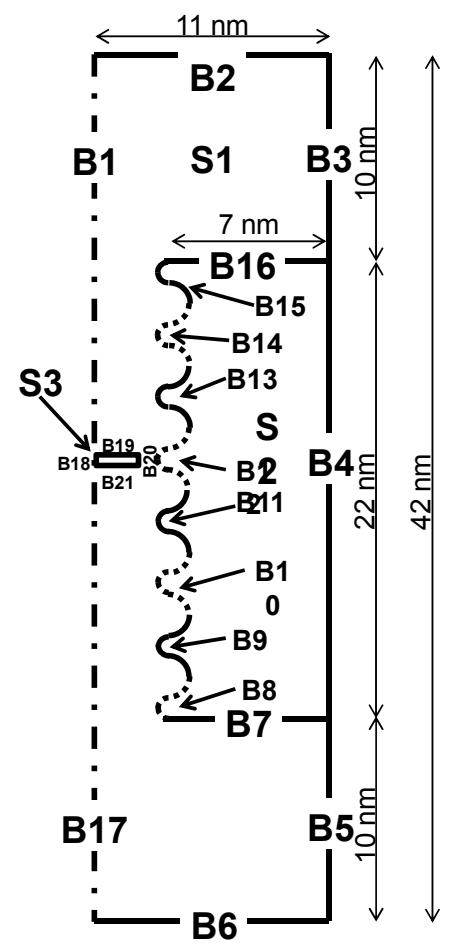

Figure 1. A) Hemi-section of the simulation space consisting of the irregularly shaped nanopore, two compartments (one on each side) and the membrane patch separating them. Rotation of the hemisection about central axis by $360^{\circ}$ generates the 3D-model. The pore at its narrowest is $3.25 \mathrm{~nm}$ and at the widest $5.5 \mathrm{~nm}$, and its length $\mathrm{L}$ is $22 \mathrm{~nm}$. The radius $\mathrm{W}$ of two compartments flanking the nanopore is $11.0 \mathrm{~nm}$. The total length of the simulation domain is $42.0 \mathrm{~nm}$. Radius of the object (a disk in this simulation) is $2.0 \mathrm{~nm}$ and its thickness is $0.5 \mathrm{~nm}$. 
Table 1. Boundary conditions

\begin{tabular}{|c|c|c|c|}
\hline Boundary & Hydrodynamics & Electrostatics & Electro-kinetics \\
\hline B1, B17 & Axial symmetry & Axial symmetry & Axial symmetry \\
\hline B2 & Pressure $\left(p_{\mathrm{u}}\right)$, no viscous stress & $\begin{array}{l}\text { Electric potential } \mathrm{V}_{\mathrm{u}} \\
\qquad(-80 \text { or }+80 \mathrm{mV})\end{array}$ & $\begin{array}{c}\mathrm{K}^{+} \text {-glutamate } \\
(150 \mathrm{mM}) \\
\mathrm{Na}^{+}-\mathrm{Cl}^{-}(0 \mathrm{mM})\end{array}$ \\
\hline B3 & Pressure $\left(p_{\mathrm{u}}\right)$, no viscous stress & $\begin{array}{l}\text { Electric potential } \mathrm{V}_{\mathrm{u}} \\
\quad(-80 \text { or }+80 \mathrm{mV})\end{array}$ & $\begin{array}{c}\mathrm{K}^{+} \text {-glutamate } \\
(150 \mathrm{mM}) \\
\mathrm{Na}^{+}-\mathrm{Cl}^{-}(0 \mathrm{mM})\end{array}$ \\
\hline B4 & Not applicable & Zero charge symmetry & Not applicable \\
\hline B5 & Pressure $\left(p_{d}\right)$ & $\begin{array}{l}\text { Electric potential } \mathrm{V}_{\mathrm{d}} \\
\qquad(0 \mathrm{mV})\end{array}$ & $\begin{array}{c}\mathrm{K}^{+} \text {-glutamate } \\
(0 \mathrm{mM}) \\
\mathrm{Na}^{+}-\mathrm{Cl}^{-}(150 \mathrm{mM})\end{array}$ \\
\hline B6 & Pressure $\left(p_{\mathrm{d}}\right)$ & $\begin{array}{l}\text { Electric potential } \mathrm{V}_{\mathrm{d}} \\
\qquad(0 \mathrm{mV})\end{array}$ & $\begin{array}{c}\mathrm{K}^{+} \text {-glutamate } \\
(0 \mathrm{mM}) \\
\mathrm{Na}^{+}-\mathrm{Cl}^{-}(150 \mathrm{mM})\end{array}$ \\
\hline B7 & No slip & Surface charge density $\sigma_{\mathrm{e}} 7$ & No flux \\
\hline B8, B10, B12, B14 & No slip & Surface charge density $\sigma_{\mathrm{e} 8}, \sigma_{\mathrm{e} 10,}, \sigma_{\mathrm{e} 12,}, \sigma_{\mathrm{e} 14}$ & No flux \\
\hline B9, B11, B13, B15 & No slip & Surface charge density $\sigma_{\mathrm{e} 9}, \sigma_{\mathrm{e} 11}, \sigma_{\mathrm{e} 13}, \sigma_{\mathrm{e} 15}$ & No flux \\
\hline B16 & No slip & Surface charge density $\sigma_{\mathrm{e} 16}$ & No flux \\
\hline B18 & Not applicable & Axial symmetry & Not applicable \\
\hline B19, B20, B21 & No slip & Surface charge density $\sigma_{\mathrm{e} 19}, \sigma_{\mathrm{e} 20,}, \sigma_{\mathrm{e} 21}$ & No flux \\
\hline
\end{tabular}

Table 2. Model parameters and constants

\begin{tabular}{|c|c|c|c|c|}
\hline Params & Values & Unit & Description & Refs \\
\hline $\mathrm{R}$ & 8.314 & $\mathrm{~J} /(\mathrm{mol} \cdot \mathrm{K})$ & Universal gas constant & [30] \\
\hline $\mathrm{T}$ & 300.0 & $\mathrm{~K}$ & Temperature & \\
\hline$\varepsilon 0$ & $8.854 \times 10^{-12}$ & $\mathrm{~F} / \mathrm{m}$ & Permittivity of vacuum & {$[30]$} \\
\hline$\varepsilon r$, Solution & 80.0 & Dimensionless & $\begin{array}{l}\text { Relative permittivity of } \\
\text { the electrolyte solution }\end{array}$ & {$[31]$} \\
\hline$\varepsilon r$,Membrane & 2.0 & Dimensionless & $\begin{array}{l}\text { Relative permittivity of } \\
\text { the membrane }\end{array}$ & {$[31]$} \\
\hline$\varepsilon r$,Object & 2.0 & Dimensionless & $\begin{array}{l}\text { Relative permittivity of } \\
\text { the object }\end{array}$ & \\
\hline$e$ & $1.602 \times 10^{-19}$ & $\mathrm{C}$ & Elementary charge & {$[30]$} \\
\hline$\overline{D_{K}}$ & $1.960 \times 10^{-9}$ & $\mathrm{~m}^{2} / \mathrm{sec}$ & $\begin{array}{l}\text { Diffusion coefficient of } \\
\mathrm{K}^{+} \text {ions }\end{array}$ & [31] \\
\hline$D_{\mathrm{Na}}$ & $1.330 \times 10^{-9}$ & $\mathrm{~m}^{2} / \mathrm{sec}$ & $\begin{array}{l}\text { Diffusion coefficient of } \\
\mathrm{Na}^{+} \text {ions }\end{array}$ & [31] \\
\hline$D_{\mathrm{cl}}$ & $2.030 \times 10^{-9}$ & $\mathrm{~m}^{2} / \mathrm{sec}$ & $\begin{array}{l}\text { Diffusion coefficient of } \\
\mathrm{Cl}^{-} \text {ions }\end{array}$ & [31] \\
\hline$D_{\text {Glutamate }}$ & $0.760 \times 10^{-9}$ & $\mathrm{~m}^{2} / \mathrm{sec}$ & $\begin{array}{l}\text { Diffusion coefficient of } \\
\text { glutamate }^{-}\end{array}$ & {$[31]$} \\
\hline$\mu$ & $1.0 \times 10^{-3}$ & $\mathrm{~Pa} \cdot \mathrm{sec}$ & Fluid viscosity & {$[30]$} \\
\hline$\rho$ & $1.0 \times 10^{3}$ & $\mathrm{~kg} / \mathrm{m}^{3}$ & Fluid density & {$[31]$} \\
\hline
\end{tabular}

Figure 1 depicts the model geometry, which comprises of 3 subdomains and 21 boundaries. Note that the protrusions and valleys are represented as small and large semi-ovals. Their minor and major axes are 1.0 and $2.0 \mathrm{~nm}$ (protrusions) and 1.5 and $3.0 \mathrm{~nm}$ (valleys; i.e., B8-B15). Table 1 lists the boundary conditions implemented in this study and Table 2 gives the parameters and constants used. Note that the Poisson equation (i.e., electrostatics) was implemented in all subdomains, while the Nernst-Plank equation (i.e., electrokinetics) and the Navier-Stokes equation (i.e., hydrodynamics) were only defined in subdomain 1 (i.e., electrolyte solution). 


\subsection{General formulation of mathematical simulations}

We used the Poisson-Nernst-Planck (PNP) equations to calculate ionic fluxes within a nanopore for all ionic species. The Poisson equation was used to compute the electrostatic potential $(\Phi)$ :

$$
-\nabla . \varepsilon_{0} \varepsilon_{r} \nabla \Phi=\rho_{e}
$$

where $\varepsilon_{0}$ is the permittivity of vacuum, $\varepsilon_{r}$ is the relative dielectric constant of solution and $\nabla$ is the gradient operator. The charge density $\rho_{e}$ (mobile and fixed) was calculated as follows:

$\rho_{\mathrm{e}}=\mathrm{F} \sum \mathrm{z}_{\mathrm{a}} \mathrm{c}_{\mathrm{a}}\left(=\mathrm{e} \sum \mathrm{z}_{\mathrm{a}} \mathrm{n}_{\mathrm{a}}\right)$

Note that $\mathrm{ca}$ is the molar concentration of each ion in $\left[\mathrm{mol} / \mathrm{m}^{3}\right], \mathrm{za}_{\mathrm{a}}$ is the valence of ion $\mathrm{a}, \mathrm{n}_{\mathrm{a}}$ is the number density of ion a and $\mathrm{F}$ is the Faraday constant $\left(9.648 \times 10^{4} \mathrm{C} / \mathrm{mol}\right)$. Note also that in these simulations the International System of Units (SI) is used, and $\mathrm{mol} / \mathrm{m}^{3}$ thus translates to $\mathrm{mmol} / \mathrm{liter}$ (or simply $\mathrm{mM}$ ). Additional factors that will influence the potential are: a) the fixed charges on the pore wall, b) the mobile charges inside the nanopore, and c) the potentials on the controlling edges.

The movement (convection-diffusion-migration) of ions in the electrolytic fluid was defined by the Nernst-Planck equation as follows:

$$
\mathbf{J}_{\mathbf{a}}=\mathbf{u c}_{\mathrm{a}}-D_{a} \nabla \mathrm{c}_{\mathrm{a}}-m_{a} \mathrm{z}_{\mathrm{a}} \mathrm{Fc}_{\mathrm{a}} \nabla \Phi
$$

where $\mathbf{J a}$ is the molar flux in $\mathrm{mol} /\left(\mathrm{m}^{2} \cdot \mathrm{s}\right)$, whereas $\mathbf{u}$ is the fluid velocity calculated by the Navier-Stokes equation. $D_{a}$ and $m_{a}$ are diffusivity and mobility of ion a, which are related by $m_{a}=D_{a} /(R T)$. Finally, $R$, and $T$ are the universal gas constant $(R=8.314 \mathrm{~J} /(\mathrm{mol} \cdot \mathrm{K})$, and absolute temperature in Kelvin, respectively.

Equation 4 gives the conservation of ionic mass in a steady state situation.

$$
\nabla . \mathbf{J}_{\mathbf{a}}=0
$$

The Navier-Stokes equation (at steady-state condition), in the presence of external forces, was applied to model fluid velocity as follows:

$$
\rho(\mathbf{u} . \nabla \mathbf{u})=-\nabla p+\nabla \cdot\left[\mu\left[\nabla \mathbf{u}+(\nabla \mathbf{u})^{T}\right]\right]+\mathbf{F}_{\mathbf{e}}
$$

$$
\nabla \cdot \mathbf{u}=0
$$

Equation (5) describes the conservation of momentum, whereas equation (6) accounts for the conservation of mass. $\rho, \mu, p$ stand for fluid density, viscosity and pressure, respectively. The vector $\mathbf{u}$ denotes the fluid velocity. Finally, $\mathbf{F}_{\mathbf{e}}$ accounts for the electric force per unit volume, calculated as: $\mathbf{F}_{\mathbf{e}}=-\rho_{e} \nabla \Phi$

\subsection{Mathematical model in the cylindrical coordinate system}

In this study we used the cylindrical coordinate system (the 2D $r-z$ plane). The velocity and its gradient in the $\theta$-direction were ignored, but there was an axial symmetry in the $z$-direction. The simplified Navier-Stokes equation in terms of components of the stress tensor $\tau$ thus reads as follows:

$r$-component: $\rho\left(\frac{\partial u}{\partial t}+u \frac{\partial u}{\partial r}+v \frac{\partial u}{\partial z}\right)=-\frac{\partial p}{\partial r}-\left[\frac{1}{r} \frac{\partial}{\partial r}\left(r \tau_{r r}\right)+\frac{\partial \tau_{r z}}{\partial z}\right]+F_{e-r}$ 
$z$-component: $\rho\left(\frac{\partial v}{\partial t}+u \frac{\partial v}{\partial r}+v \frac{\partial v}{\partial z}\right)=-\frac{\partial p}{\partial z}-\left[\frac{1}{r} \frac{\partial}{\partial r}\left(r \tau_{r z}\right)+\frac{\partial \tau_{z z}}{\partial z}\right]+F_{e-z}$

where $u$ and $v$ are the $r$ - and $z$-components of the fluid velocity, respectively. $\tau_{i j}$ is also the $i j$-th component of the viscous stress tensor $\tau$, which for the Newtonian fluids in 2D $r$-z plane (in the cylindrical coordinate system) are defined as:

$$
\begin{aligned}
& \tau_{r r}=-\mu\left[2 \frac{\partial u}{\partial r}-\frac{2}{3}(\nabla \cdot \mathbf{u})\right] \\
& \tau_{z z}=-\mu\left[2 \frac{\partial v}{\partial z}-\frac{2}{3}(\nabla \cdot \mathbf{u})\right] \\
& \tau_{r z}=-\mu\left[\frac{\partial v}{\partial r}+\frac{\partial u}{\partial z}\right]
\end{aligned}
$$

where $\nabla \cdot \mathbf{u}=\frac{1}{r} \frac{\partial}{\partial r}(r u)+\frac{\partial v}{\partial z}$

As mentioned above the electrostatic force is defined as $\mathbf{F}_{\mathbf{e}}=-\rho_{e} \nabla \Phi$. Note that the gradient operator in the cylindrical coordinate system is given by:

$[\nabla \Phi]_{r}=\frac{\partial \Phi}{\partial r}, \quad[\nabla \Phi]_{z}=\frac{\partial \Phi}{\partial z}$

With a substitution of the components of the stress tensor given in equations 9-13, into equations 7 and 8 with constants $\rho$ and $\mu$, we obtained equations 14 and 15 describing the Navier-Stokes equation in terms of velocity gradients.

$r$-component: $\rho\left(\frac{\partial u}{\partial t}+u \frac{\partial u}{\partial r}+v \frac{\partial u}{\partial z}\right)=-\frac{\partial p}{\partial r}+\mu\left[\frac{\partial}{\partial r}\left(\frac{1}{r} \frac{\partial}{\partial r}(r u)\right)+\frac{\partial^{2} u}{\partial z^{2}}\right]-\rho_{c} \frac{\partial \Phi}{\partial r}$

$z$-component: $\rho\left(\frac{\partial v}{\partial t}+u \frac{\partial v}{\partial r}+v \frac{\partial v}{\partial z}\right)=-\frac{\partial p}{\partial z}+\mu\left[\frac{1}{r} \frac{\partial}{\partial r}\left(r \frac{\partial v}{\partial r}\right)+\frac{\partial^{2} v}{\partial z^{2}}\right]-\rho_{c} \frac{\partial \Phi}{\partial z}$

Finally, the divergence operator in equations (1) and (4) was defined as:

$\nabla . \mathbf{J}=\frac{1}{r} \frac{\partial}{\partial r}\left(r J_{r}\right)+\frac{\partial J_{z}}{\partial z}$

where $J_{r}$ and $J_{z}$ are the $r$ - and $z$-components of vector $\mathbf{J}$.

\subsection{Calculation of forces acting on the object}

The dielectric force acting on the object can be obtained by evaluating the Maxwell stress tensor on the boundary between the object and the solution. On a given boundary the Maxwell stress tensor (S; a coordinate independent tensor) was defined as follows:

$$
\mathbf{S}=\varepsilon_{r} \varepsilon_{0}\left[\mathbf{E} \mathbf{E}-\frac{1}{2}(\mathbf{E}[\mathbf{E}) \mathbf{I}]\right.
$$

As already stated $\varepsilon_{s}$ is the relative dielectric constant of solution and $\varepsilon_{o}$ is the relative dielectric constant of the object, i.e., of two subdomains on two sides of the horizontal boundaries B19 and B21 
(Figure 1), whereas $\varepsilon_{0}$ is the permittivity of vacuum. $\mathbf{E}$ is the vector of the electric filed $\left(E_{r}, E_{z}\right)^{\mathrm{T}}$, and $\mathbf{I}$ is the identity tensor.

The force, $\mathbf{F}_{\mathbf{M}}=\left(F_{M r}, F_{M z}\right)^{\mathrm{T}}$ due to the Maxwell stress tensor on the horizontal boundaries

B19 or B21; (i.e., FM19 or FM21 respectively) were defined as follows:

$$
\mathbf{F}_{\mathbf{M}}=\int_{\Omega} \mathbf{n} \mathbf{S} d A=\int_{\partial \Omega} \mathbf{n} \mathbf{S}(2 \pi r) d r
$$

where $\Omega$ stands for the horizontal surfaces of the object whose edges are B19 or B21 and revolves around the $z$-axis; $\mathbf{n}$ denotes the outward unit normal on a given surface, and $d A$ accounts for the differential surface area element. $\partial \Omega$ stands for a horizontal boundary of the object (i.e., B19 or B21); $d r$ is the differential boundary element, and $r$ stands for the radius of the object.

In addition to the forces due to the Maxwell stresses in the electrolyte media, charged surfaces exert the Coulomb force:

$$
\mathbf{F}_{\sigma}=\int_{\Omega} \sigma_{\mathrm{e}} \mathbf{E} d A=\int_{\partial \Omega} \sigma_{\mathrm{e}} \mathbf{E}(2 \pi r) d r
$$

where $\sigma_{\mathrm{e}}$ is the surface charge density corresponding to the boundaries B19 or B21, that is $\sigma_{\mathrm{e} 19}$ or $\sigma_{\mathrm{e} 21}$, respectively.

The other force acting on the upper and lower surfaces of the object is due to the hydrodynamic pressure acting on these surfaces.

$$
\mathbf{F}_{\mathbf{p}}=\int_{\Omega} p d A=\int_{\partial \Omega} p(2 \pi r) d r
$$

The total force $\mathbf{F}_{\mathbf{t}}$ is the summation of the forces $\mathbf{F}_{\mathbf{M}}, \mathbf{F}_{\mathbf{\sigma}}$, and $\mathbf{F}_{\mathbf{p}}$ acting on the boundaries B19 or B21 that is $F_{t 19}$ or $F_{221}$, respectively. Note that for simplicity we omitted the subscripts 19 and 21 in $\mathbf{F}_{\mathbf{M}}, \mathbf{F}_{\mathbf{\sigma}}$, $\mathbf{F}_{\mathbf{p}}$, and $\mathbf{F}_{\mathrm{t}}$.

The system of coupled Poisson-Nernst-Planck and Navier-Stokes equations was solved using a finite element method based on a commercial software package program COMSOL Multiphysics 4.3 (COMSOL, Burlington, MA, USA), and the postprocessing was performed using a software package for scientific and engineering computation MATLAB (MathWorks, Natick, MA, USA).

\section{Results}

\subsection{Positively charged object opposite negatively charged pore wall protrusion}

This study is an attempt to understand how a charged object (a disk whose radius is $2 \mathrm{~nm}$ and thickness $0.5 \mathrm{~nm}$ ) would move axially, and rotate in a charged irregular nanopore. A nanopore is considered as irregular if its radius is not constant, but changes in a periodic manner (see Figure 1). In an uniformly charged object the charge density $\left(\sigma_{\circ}\right)$ on all surfaces was $+64 \mathrm{mC} / \mathrm{m}^{2}$, but in some cases we considered non-uniformly charged objects where only the upper surface was charged with the same $\sigma_{\mathrm{o}} . \sigma_{\mathrm{p}}$ on all protrusions of the nanopore was the same $\left(64 \mathrm{mC} / \mathrm{m}^{2}\right)$, but the sign changed in an alternating manner, and the change in each case occurred in the middle of each valley. The voltage on the upper controlling surfaces $\left(\mathrm{V}_{\mathrm{u}}\right)$ was typically $-80 \mathrm{mV}$ (but in some cases it was $+80 \mathrm{mV}$; see below). 

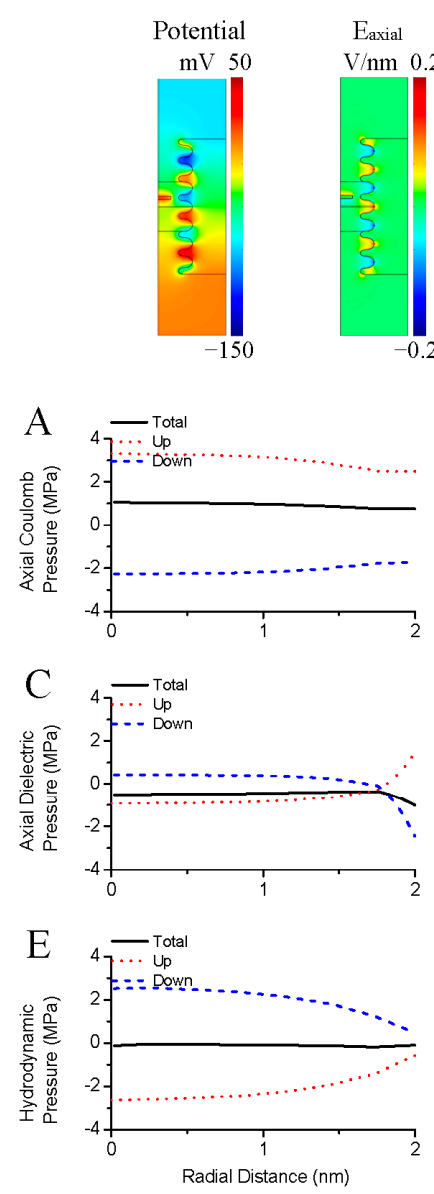
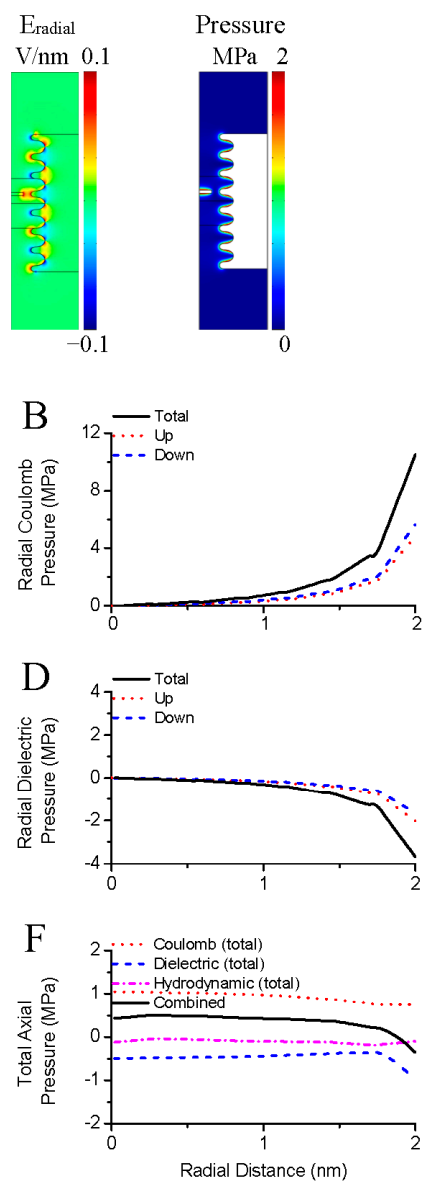

Figure 2. A positively charged object positioned opposite a negatively charged protrusion of an irregular nanopore, and with a negative upward electric field (i.e., the potential at the upper controlling edges $\left(\mathrm{V}_{\mathrm{u}}\right)$ was $\left.-80 \mathrm{mV}\right)$, would move upwards and rotate clockwise. A) The color coded 2D distributions of the potential, electric field (axial and radial) and hydrodynamic pressure are shown on the top. The calibration bars are as indicated. A) The axial Coulomb pressure at the upper and lower edges of the object and the total axial Coulomb pressure of the object. B-E) The corresponding radial Coulomb pressures, axial dielectric pressures, radial dielectric pressures and the axial hydrodynamic pressures. F) The total axial pressures - Coulomb, dielectric, hydrodynamic and their sum (i.e., combined pressure). The potential at the lower controlling edges $\mathrm{V}_{\mathrm{d}}$ was $0 \mathrm{mV}$. The pressure at the upper $\left(\mathrm{p}_{\mathrm{u}}\right)$ and at the lower $\left(\mathrm{p}_{\mathrm{d}}\right)$ controlling edges was $0 \mathrm{~Pa}$. The charge density on the pore wall protrusions alternated between $+64 \mathrm{mC} / \mathrm{m}^{2}$ and $-64 \mathrm{mC} / \mathrm{m}^{2} . \mathrm{K}^{+}$and glutamate ${ }^{-}$ concentrations were $150 \mathrm{mM}$ and $\mathrm{Na}^{+}$and $\mathrm{Cl}^{-}$concentrations were $0 \mathrm{mM}$ at the upper controlling edges, and the reverse was at the lower controlling edges.

Figure 2 shows color coded 2D distributions of the potential, axial and radial electric fields and hydrodynamic pressure (top panels) for the object positioned opposite negatively charged protrusion. Figure 2A gives the radial profiles of the axial Coulomb pressure at the upper surface $\left(\mathrm{CP}_{\mathrm{ax}, \mathrm{u}}\right)$, which is positive (i.e., pointing upwards). At the lower surface $\left(\mathrm{CP}_{\mathrm{ax}, \mathrm{d}}\right)$ it is negative (i.e., pointing downwards). Both $\mathrm{CP}_{\mathrm{ax}, \mathrm{u}}$ and $\mathrm{CP}_{\mathrm{ax}, \mathrm{d}}$ diminish in amplitude with radial distance, albeit modestly. As they differ in amplitude the total axial Coulomb pressure $\left(\mathrm{CP}_{\mathrm{ax}, \mathrm{tot}}\right)$ is non-zero, and $\mathrm{CP}_{\mathrm{ax}, \mathrm{u}}$ being greater it is positive. Figure $2 \mathrm{~B}$ shows the radial profiles of the upper and lower radial Coulomb pressures ( $\mathrm{CP}_{\text {rad,u }}$ and $\mathrm{CP}$ rad,d), and their sum (i.e., the total radial Coulomb pressure or $\left.\mathrm{CP}_{\text {rad,tot }}\right)$. $\mathrm{CP}_{\text {rad,u }}$ and $\mathrm{CP}_{\text {rad,d }}$ were near zero value at small radial distances, and both rose as the radial distance increased. Near the end of the object radial Coulomb pressures were significantly larger than corresponding axial Coulomb pressures. Given that in this study $\sigma_{o}$ on all charged surfaces was 
constant, and that Coulomb pressure (axial or radial) is a product of the electric field (axial or radial component) and $\sigma_{\mathrm{o}}$, radial profiles of the Coulomb pressure also showed how the electric filed changed in each case.

The axial dielectric pressures (DPax,u and DPax,d; Figure 2C) were considerably smaller and in each case pointed in the direction opposite of the corresponding Coulomb pressures (i.e., negative at the upper surface and positive at the lower surface). They also diminished in amplitude as the radial distance increased, but eventually they also changed the sign. Nevertheless, note that though $\mathrm{CP}_{\text {ax,tot }}$ was greater than $\mathrm{DP}_{\text {ax,tot }}$ they differred less than the corresponding axial pressures at each surface. The radial dielectric pressures ( $\mathrm{DP}_{\mathrm{rad}, \mathrm{u}}$ and $\mathrm{DP}$ rad,d) are (as are $\mathrm{CP}_{\text {rad,u }}$ and $\mathrm{CP}_{\mathrm{rad}, \mathrm{d}}$ ) near zero at small radial distances, but became negative as the radial distance rose (Figure 2D).

The hydrodynamic wall pressures $\mathrm{Pw}_{\mathrm{u}}$ and $\mathrm{Pwd}_{\mathrm{d}}$ were similar in value but of opposite sign at two surfaces. Although they diminished significantly as the radial distance increased, the total hydrodynamic wall pressure ( $\left.\mathrm{PW}_{\mathrm{tot}}\right)$ was near zero regardless of radial distance. (Figure 2E). Note that the hydrodynamic pressure is a scalar and both surfaces are flat. The direction of the hydrodynamic wall pressure is thus defined by a surface vector. There is thus no radial hydrodynamic wall pressure (i.e., the hydrodynamic wall pressure always refers to the axial hydrodynamic wall pressure).

Figure $2 \mathrm{~F}$ gives combined axial pressure (i.e., the sum of all three total axial pressures - $\mathrm{CP}_{\mathrm{ax}, \mathrm{tot}}$ $\mathrm{DP}_{\mathrm{ax}, \mathrm{tot}}$ and $\left.\mathrm{PW}_{\mathrm{tot}}\right)$, which was positive over most of the object, diminished as the radial distance increased becoming negative at the end of the object. Given that the integral of the total force was negative (its value was $-0.091 \mathrm{pN}$ ), the object would move downwards. As the radial distance profile of the total axial pressure shows the object would rotate clockwise. It would move towards the wall of the nanopore, because the radial Coulomb force was positive, and larger than the radial dielectric force, which opposed it.

\subsection{Positively charged object opposite nanopore valley}

The object was placed opposite valley formed by two protrusions having the same $\sigma_{\mathrm{p}}$, but of opposite sign. Figure 3 (top panels) gives color coded 2D distributions of the potential, axial and radial electric fields and hydrodynamic pressure. The axial and radial Coulomb pressures did not differ greatly from those when the object was positioned opposite negatively charged protrusion, but differences did exist. $\mathrm{CP}$ ax,tot remained positive, but rose although very modestly with radial distance

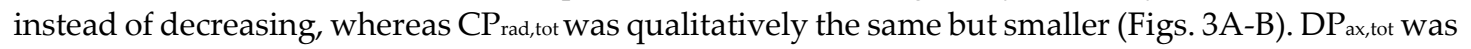
qualitatively the same, but was more negative (Figure 3C), and DPrad,tot changed very little (Figure 3D). Combined radial Coulomb and dielectric pressures would again move the object towards the wall. They were opposed by a much smaller hydrodynamic pressure on the disk radial surface (not shown but can be inferred from the values of the axial hydrodynamic pressures near the surface; see below). Pwu and P $\mathrm{wd}_{\mathrm{d}}$ also changed very little, and $\mathrm{Pw}_{\text {tot }}$ remained near zero (Figure 3E). The combined axial pressure nevertheless changed qualitatively. It was lower over most of the radial distance, but rose modestly instead of decreasing near the end of the object. As a result, the object would not move downwards, but upwards (the integral of the total force on the object was positive; its value was $+0.069 \mathrm{pN}$ ), and would rotate not clockwise but counter-clockwise. 

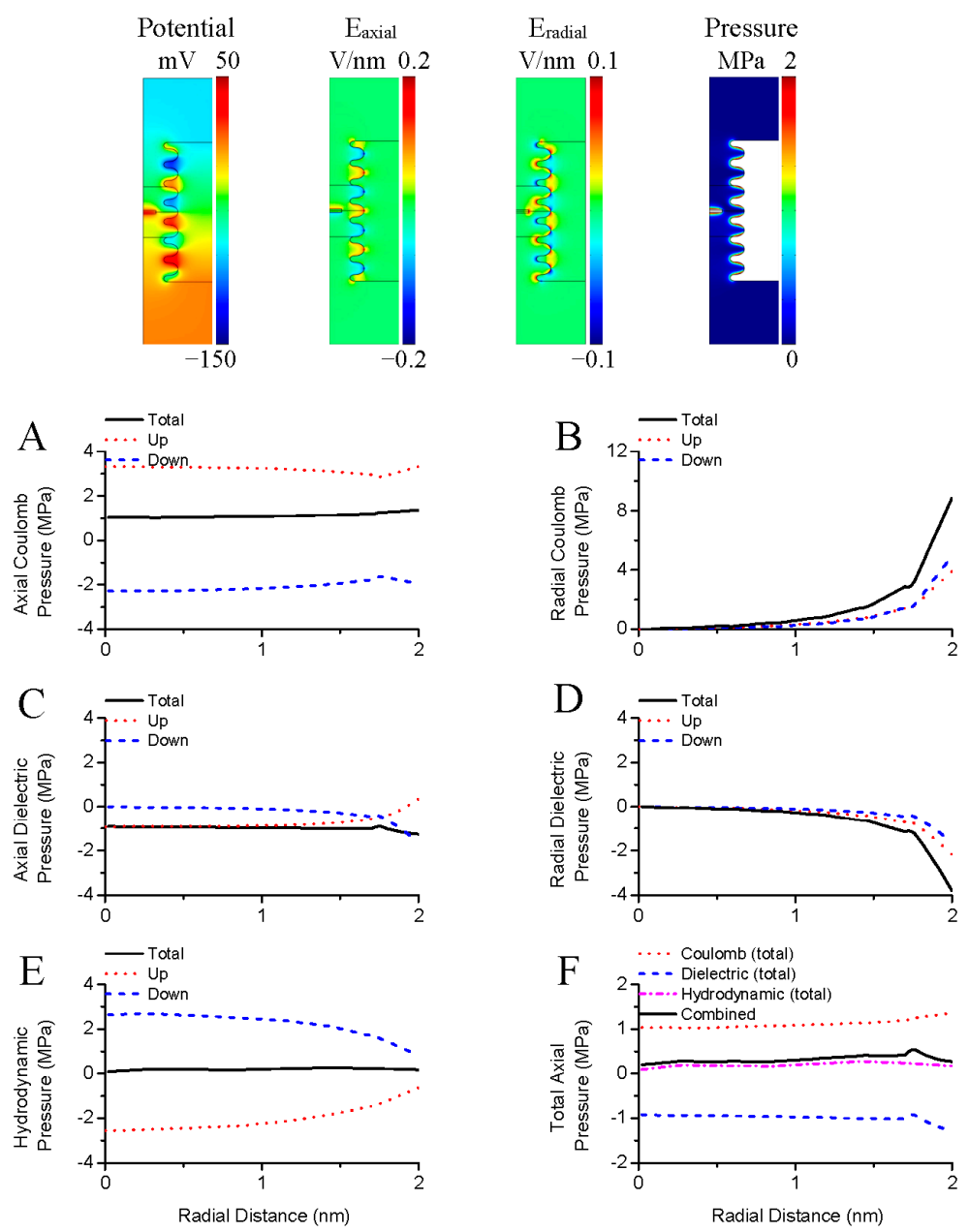

Figure 3. A positively charged object positioned in between a negatively charged and a positively charged pore protrusion of an irregular nanopore, and with a negative upward electric field shows a very modest tendency to move upwards and to rotate counter-clockwise. Top panels show the color coded 2D distributions of the potential, electric field (axial and radial) and hydrodynamic pressure. The calibration bars are as indicated. A) The axial Coulomb pressure at the upper and lower edges of the object and the total axial Coulomb pressure of the object. B-E) The corresponding radial Coulomb pressures, axial dielectric pressures, radial dielectric pressures, and the axial hydrodynamic pressures. F) The total axial pressures - Coulomb, dielectric, hydrodynamic and their sum (i.e., combined pressure). $V_{d}=0 \mathrm{mV}$, whereas $p_{u}$ and $p_{d}$, charge density on the pore wall protrusions and ion (and glutamate ${ }^{-}$) concentrations at the upper and lower controlling edges were as in Figure 2. 


\subsection{Positively charged object opposite positively charged protrusion}
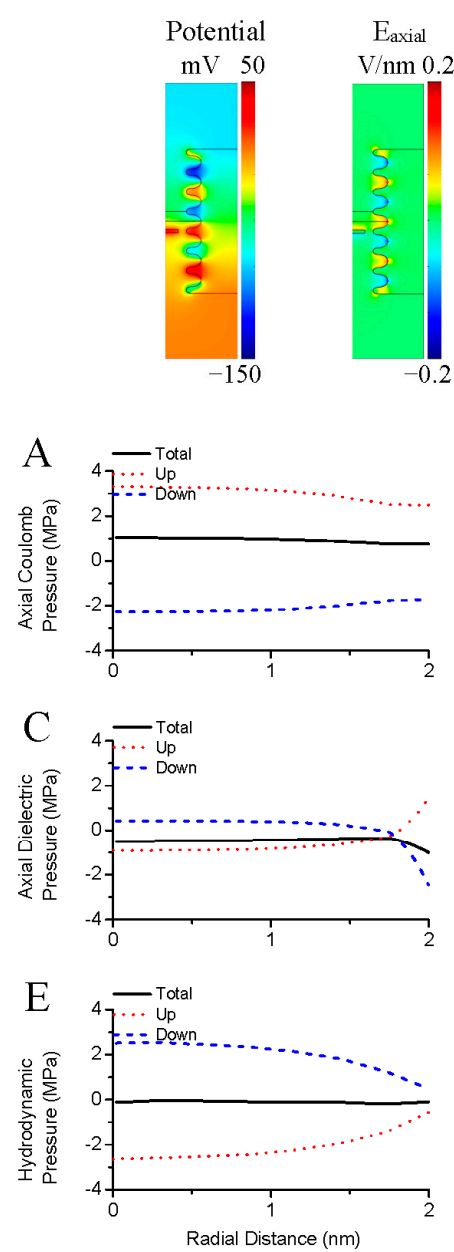
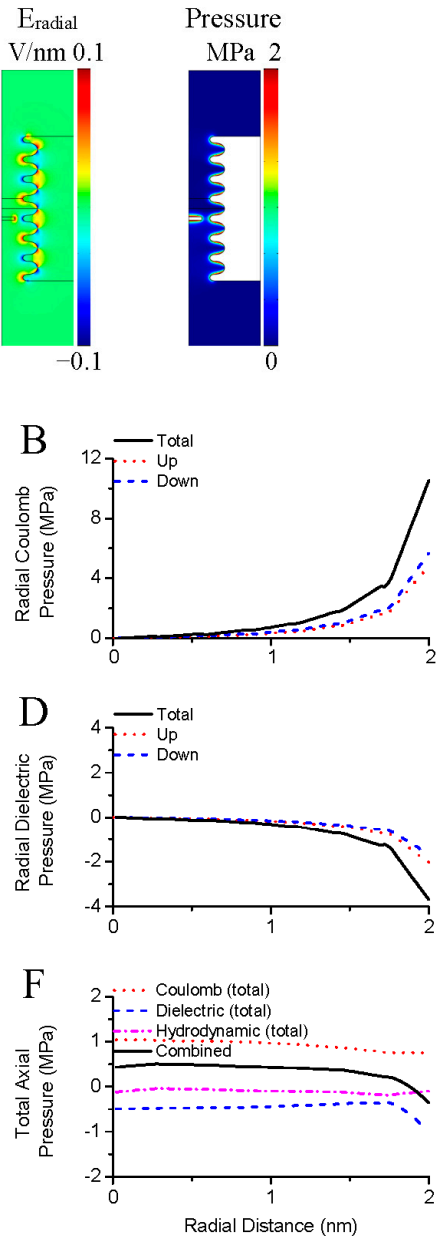

Figure 4. A positively charged object positioned opposite a positively charged protrusion of an irregular nanopore (with a negative upward electric field), would move upwards and rotate clockwise. The panels on the top are color coded 2D distributions of the potential, electric field (axial and radial) and hydrodynamic pressure. The calibration bars are as indicated. A) The axial Coulomb pressure at the upper and lower edges of the object and the total axial Coulomb pressure of the object. B-E) The corresponding radial Coulomb pressures, axial dielectric pressures, radial dielectric pressures and axial hydrodynamic pressures. F) The total axial pressures - Coulomb, dielectric, hydrodynamic and their sum (i.e., combined pressure). $\mathrm{V}_{\mathrm{d}}=0 \mathrm{mV}, \mathrm{p}_{\mathrm{u}}$ and $\mathrm{p}_{\mathrm{d}}$, charge density on the pore wall protrusions and the ion (and glutamate ${ }^{-}$) concentrations at the upper and lower controlling edges were as in Figure 2.

Figure 4 top panels depict the color coded 2D distributions of the potential, electric field (axial and radial), and hydrodynamic pressure for a charged object opposite positively charged protrusion, and with $\mathrm{V}_{\mathrm{u}}=-80 \mathrm{mV}$. Most radial distance profiles resembled those with object facing the negative protrusion. The following are the differences. Positive $\mathrm{CP}_{\mathrm{ax}, \mathrm{u}}$ and negative

$\mathrm{CP}_{\mathrm{ax}, \mathrm{d}}$ yield $\mathrm{CP}_{\mathrm{ax}, \text { tot }}$ that remained positive overall and independent of the radial distance (Figure

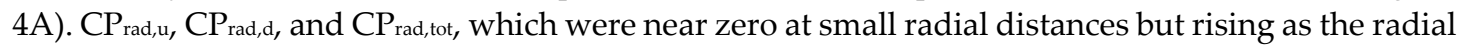
distance increased, were smaller than those when the object was facing the negative protrusion (Figure 4B). DPrad, and DPrad,d (Figs. 4C-4D) were marginally smaller near the end of the object. DPax,tot (negative and comparatively small) diminished marginally, whereas DPrad,tot was very similar to that in Figure 2. Finally, Pwu and Pwd changed little, and Pwtot remained near zero regardless of radial distance (Figure 4E). When $\mathrm{CP}_{\mathrm{ax}, \mathrm{tot}}, \mathrm{DP}_{\mathrm{ax}, \mathrm{tot}}$, and $\mathrm{PW}_{\text {tot }}$ were added, the combined pressure depended 
on the radial distance similarly to when the object was facing the negatively charged protrusion. The object would rotate clockwise and move downwards, but far less quickly. The integral of the total force on the object was negative, but much smaller (its value was $-0.011 \mathrm{pN}$ ).

\subsection{Reversal of the electric field}
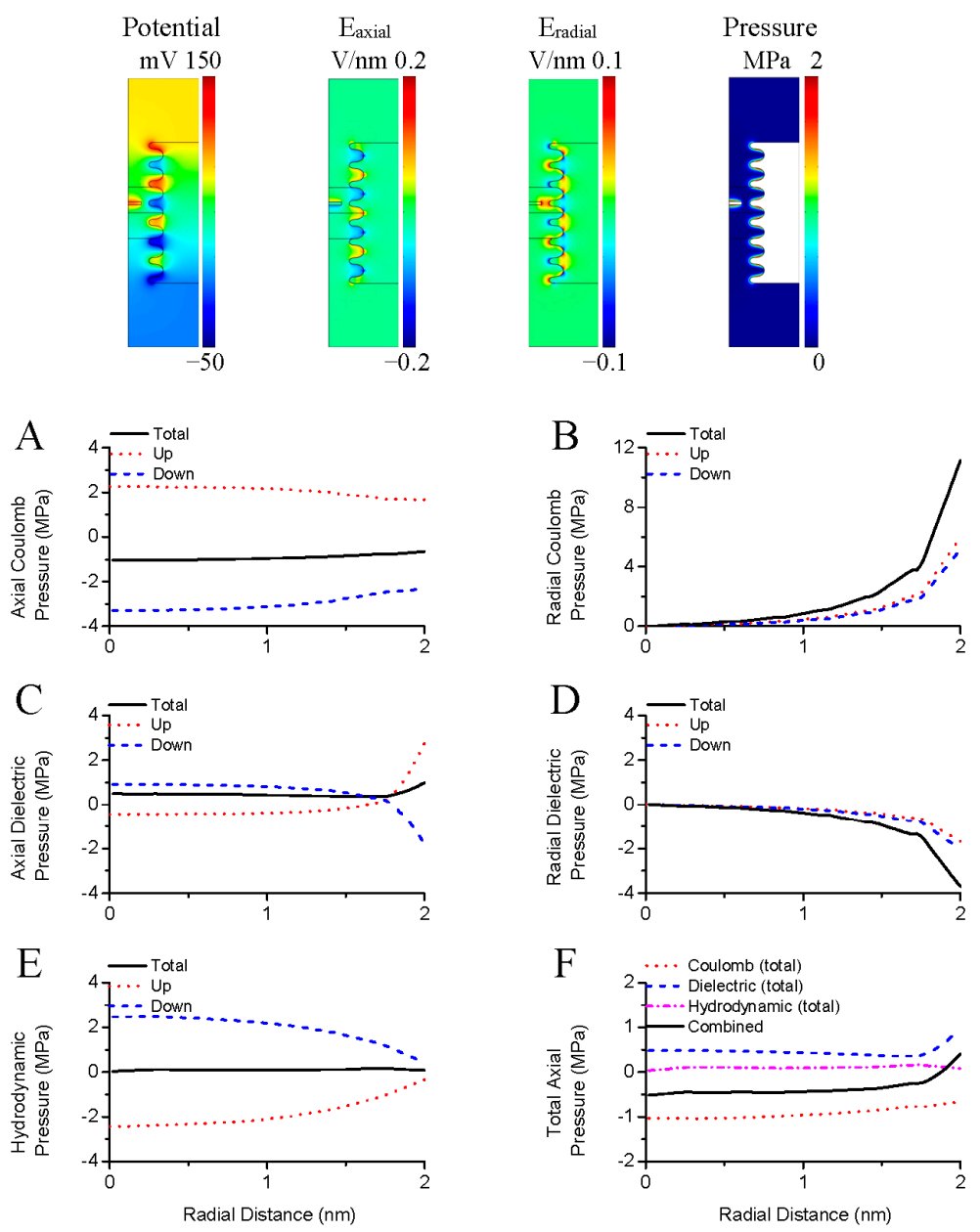

Figure 5. A positively charged object positioned opposite a negatively charged protrusion of an irregular nanopore, but with a positive electric field (i.e., $\mathrm{V}_{\mathrm{u}}=+80 \mathrm{mV}$ ), would move downwards and rotate counter-clockwise. A) The color coded 2D distributions of the potential, electric field (axial and radial) and hydrodynamic pressure are given on the top. The calibration bars are as indicated. A) The axial Coulomb pressure at the upper and lower edges of the object and the total axial Coulomb pressure of the object. B-E) The corresponding radial Coulomb pressures, axial dielectric pressures, radial dielectric pressures and axial hydrodynamic pressures. F) The total axial pressures - Coulomb, dielectric, hydrodynamic and their sum (i.e., combined pressure). $\mathrm{V}_{\mathrm{d}}=0 \mathrm{mV}, \mathrm{p}_{\mathrm{u}}$ and $\mathrm{p}_{\mathrm{d}}$, charge density on the pore wall protrusions and ion (and glutamate ${ }^{-}$) concentrations at the upper and lower controlling edges were as in Figure 2.

Figure 5 shows the electrostatic variables when the external electric field is reversed (i.e., if $V_{u}$ is $+80 \mathrm{mV}$ instead of $-80 \mathrm{mV}$ ). The object was positively charged and was opposite negatively charged protrusion (as in Figure 2). Top panels are color coded 2D distributions of the potential, axial and radial electric field and hydrodynamic pressure. The reversal of the external electric field rendered $\mathrm{CP}_{\mathrm{ax}, \mathrm{u}}$ and $\mathrm{CP}_{\mathrm{ax}, \mathrm{d}}$ more negative and $\mathrm{CP}_{\mathrm{ax}, \mathrm{tot}}$ became negative instead of being positive (Figure 5A). CPrad,u, CPrad,d, and CPrad,tot changed little (Figure 5B). DPax,u (which was negative) became less negative, whereas $\mathrm{DP}_{\mathrm{ax}, \mathrm{d}}$ (which was positive) became more positive, and as a result, DPax,tot became 
positive, but near the end of the object both changed the sign (Figure 5C). In contrast, DPrad,u, DPrad,d, and DPrad,tot (Figure 5D), and Pwu, Pwu and Pwtot changed very little (Figure 5E). Figure 5F gives all total pressures. The combined pressure was greatly influenced by the reversal of the external electric field. Instead of being positive for most of the radial distances it was now negative, and instead of decreasing near the end it increased. The object will clearly rotate counter-clockwise. It moved upwards, as the integral of the total force on the object was positive, and comparatively large (its value was $+0.103 \mathrm{pN})$.

\subsection{Partly charged object}
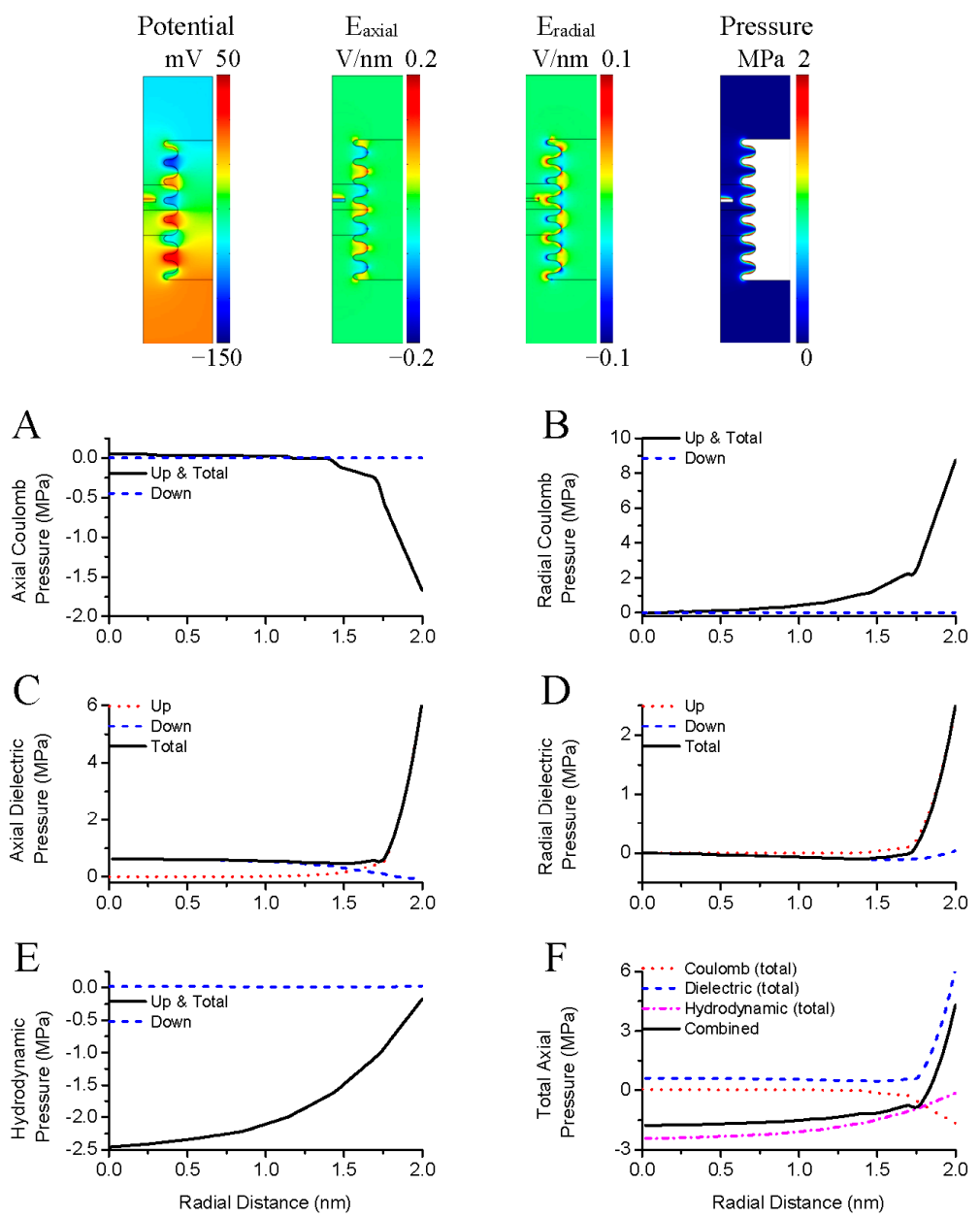

Figure 6. A positively but partially charged object (only the upper surface is charged; $\sigma_{o}=+64 \mathrm{mC} / \mathrm{m}^{2}$ ) positioned opposite a negatively charged protrusion of an irregular nanopore with a negative upward electric field $\left(\mathrm{V}_{\mathrm{u}}=-80 \mathrm{mV}\right)$ would move very rapidly downwards, and would rotate counter-clockwise. A) Top panels depict color coded 2D distributions of the potential, electric field (axial and radial) and hydrodynamic pressure. The calibration bars are as indicated. A) The axial Coulomb pressure at the upper and lower edges of the object, and the total axial Coulomb pressure of the object. B-E) The corresponding radial Coulomb pressures, axial and radial dielectric pressures, and axial hydrodynamic pressures. F) The total axial pressures - Coulomb, dielectric, hydrodynamic and their sum (i.e., combined pressure). $\mathrm{V}_{\mathrm{d}}=0 \mathrm{mV}$, whereas $\mathrm{p}_{\mathrm{u}}$ and $\mathrm{p}_{\mathrm{d}}$, charge density on the pore wall protrusions, and the ion (and glutamate ${ }^{-}$) concentrations at the upper and lower controlling edges were as in Figure 2.

Figure 6 gives the color coded 2D distributions of the potential, electric field and hydrodynamic pressure for a partly charged object $\left(\sigma_{o}=+64 \mathrm{mC} / \mathrm{m}^{2}\right.$ on the upper surface) opposite negatively charged 
protrusion, and with $\mathrm{V}_{\mathrm{u}}=-80 \mathrm{mV}$. The radial distance profiles of various pressures on the object differred significantly from those when it faced the negatively charged protrusion, but was uniformly charged. In this case $\mathrm{CP}_{\mathrm{ax}, \mathrm{u}}$, which was marginally positive near the pore center, became clearly negative near the end of the object, whereas $\mathrm{CP}_{\mathrm{ax}, \mathrm{d}}$ was zero. Note that $\mathrm{CP}_{\mathrm{ax}, \mathrm{u}}$ and $\mathrm{CP}$ ax,tot were identical in this case (Figure 6A). The radial Coulomb pressures also differred significantly. CPrad,u was qualitatively similar but bigger, whereas $\mathrm{CP}_{\text {rad,d }}$ was zero. As a result, $\mathrm{CP}_{\text {rad,tot }}$ was smaller (Figure 6B). $\mathrm{DP}_{\mathrm{ax}, \mathrm{d}}$ was quanitatively similar to DPax,d when the object was uniformly charged, but DPax,u was much greater near the end of the object (Figure 6C). The radial dielectric pressures on both surfaces were qualitatively and quantitatively different, being positive near the end of the object, especially on the upper surface. DP rad,tot thus became positive near the object's end (Figure 6D). Pwu changed little, but Pwd was zero, and as a result Pwtot was negative, but diminishing in value as the radial distance

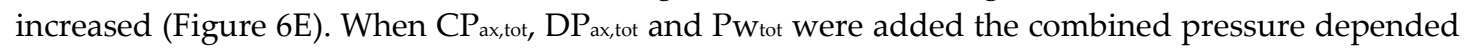
on the radial distance very differently. Near the center until almost the end of the object the combined pressure was negative, but increased becoming quite positive at the end of the object. This object would thus also move downwards, but far more quickly. The integral of the total force on the object was negative, but it was far greater (its value was $-8.618 \mathrm{pN}$ ). Moreover, the object would rotate counter-clockwise.

\subsection{Axial water velocity}

Figure 7 depicts axial water velocity (Vwax) for an object that was either uniformly charged object positioned opposite negatively charged protrusion (A), between two oppositely charged protrusions (B), and opposite a positively charged protrusion (C) with $\mathrm{V}_{\mathrm{u}}=-80 \mathrm{mV}$. In the fourth case, $\mathrm{V}_{\mathrm{u}}$ was +80 $\mathrm{mV}$, and the object was opposite negatively charged protrusion (D). Finally, we give axial water velocity for a partially charged object $\left(\sigma_{0}=+64 \mathrm{mC} / \mathrm{m}^{2}\right.$ at the upper surface, but $\sigma_{\mathrm{o}}=0 \mathrm{mC} / \mathrm{m}^{2}$ at other surfaces) opposite negatively charged protrusion, but $\mathrm{V}_{\mathrm{u}}=-80 \mathrm{mV}(\mathrm{E})$, or $\mathrm{V}_{\mathrm{u}}=+80 \mathrm{mV}(\mathrm{F})$. The color coded 2D distributions on the top show how the pore irregularity, the object position and charging and external electric field influence Vwax. The effect was further quantified at $\mathrm{r}=1 \mathrm{~nm}$ (i.e., at the half disk radius) and at $\mathrm{r}=2.5 \mathrm{~nm}$ (i.e., in between the object's end and the maximal protrusion of the nanopore).

VWax varied axially more near the pore center, but all its maxima and minima were opposite the protrusions (i.e., they alternated; Figs. 7A-D). Moreover, a downward movement near one protrusion can be followed by an upward movement near the next protrusion (Figs. 7A and 7D-F). The maxima and minima loci at $\mathrm{r}=2.5 \mathrm{~nm}$ and at $\mathrm{r}=1 \mathrm{~nm}$ did not typically coincide (i.e., their axial positions were not necessarily the same), and the maxima at $\mathrm{r}=1 \mathrm{~nm}$ may be associated with the minima at $\mathrm{r}=2.5 \mathrm{~nm}$. The locations of the VWax maxima or minima were not affected greatly by the position of the object, but their amplitudes changed. The external electric field had a significant effect. If $\mathrm{V}_{\mathrm{u}} \mathrm{was}-80 \mathrm{mV}$, and if the object was opposite negatively charged protrusion the water moved in both directions, but the downward movement was greater (Figure 7A). With object positioned opposite the valley, the water moved exclusively downwards, and Vwax variations at $\mathrm{r}=2.5 \mathrm{~nm}$ became less pronounced (Figure 7B). With positively charged protrusion the pattern of Vwax was similar to that with negatively charged protrusion, but $\mathrm{VW}_{\text {ax }}$ changed more at $\mathrm{r}=2.5 \mathrm{~nm}$ (Figure $7 \mathrm{C}$ ). When the external electric field reversed, the direction of the water movement reversed. In this case, it moved predominantly upwards, but its peaks were similarly positioned (Figure 7D). If the partially charged object was positioned opposite negatively charged protrusion and $\mathrm{V}_{\mathrm{u}}=-80 \mathrm{mV}$ (as in Figure 7A), the maxima and minima were similar in value, but opposite in sign (Figure $7 \mathrm{E}$ ). If $\mathrm{V}_{\mathrm{u}}=+80 \mathrm{mV}$, the position of minima and maxima was reversed (Figure 7F). 

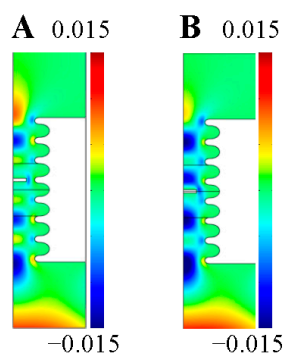

Axial Velocity $(\mathrm{m} / \mathrm{sec})$
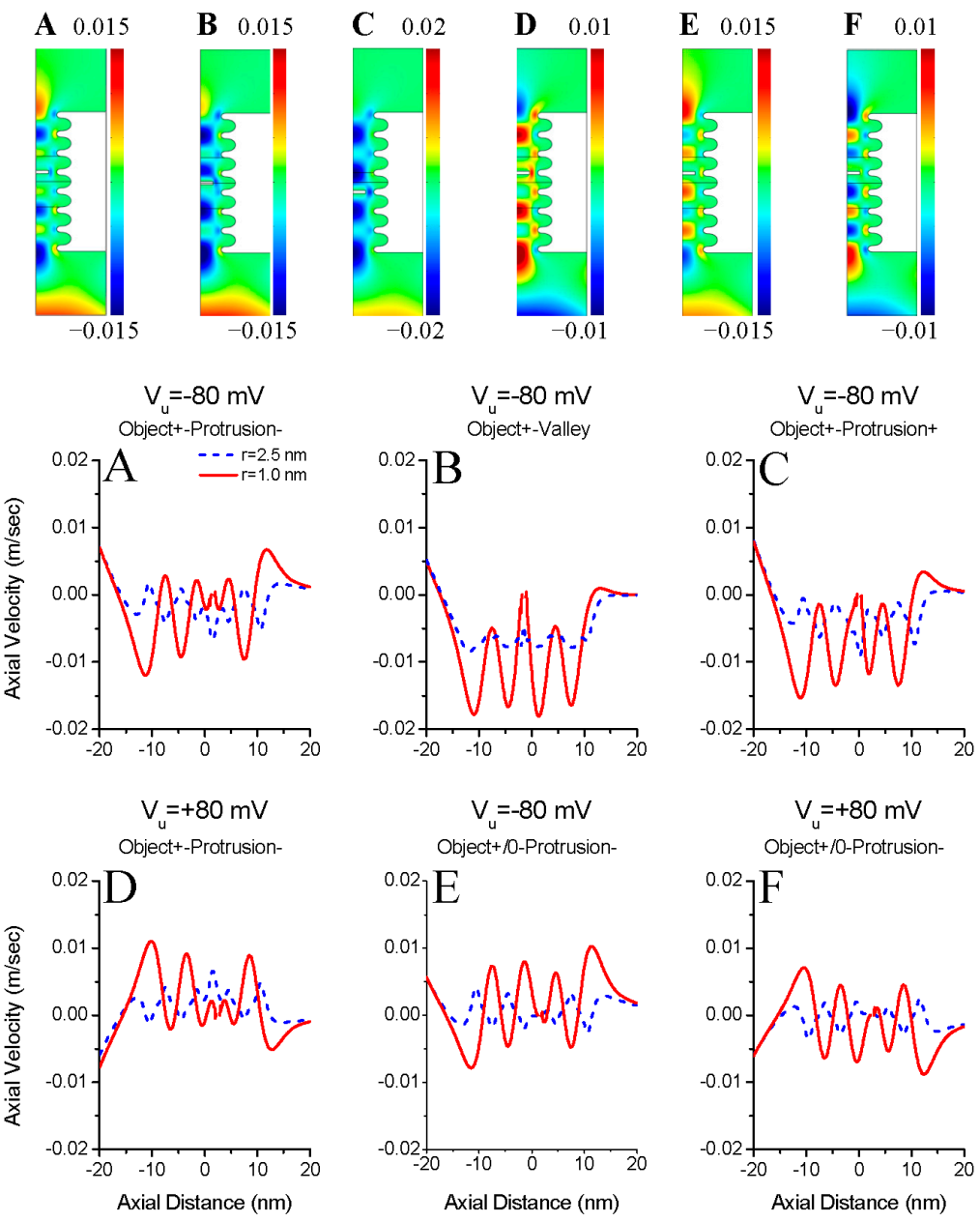

Figure 7. The velocity of the axial water movement changes significantly along the nanopore, and both velocity maxima and minima (or in some cases the upward and downward velocity peaks) occur at the level of protrusions. If $\mathrm{V}_{\mathrm{u}}=-80 \mathrm{mV}$, and positively charged object is positioned opposite a negatively charged protrusion (A), between two oppositely charged protrusions (B), and opposite a positively charged protrusion $(C)$ the velocity profiles change, but remain qualitatively the same. If $\mathrm{Vu}_{\mathrm{u}}$ becomes $+80 \mathrm{mV}$, the direction of water movement changes (the object is as in A opposite a negatively charged protrusion (D). Partial charging of the object (the upper surface charged, but not the rest) does not alter qualitatively axial water flow regardless of the $V_{u}$ sign (Figure 7E-F). The color coded $2 \mathrm{D}$ distributions on the top give the axial water velocity. A-F) The axial water velocity at $\mathrm{r}=1$ $\mathrm{nm}$ (i.e., at the half disk radius; red solid line) and at $\mathrm{r}=2.5 \mathrm{~nm}$ (i.e., in between the object's end and the maximal protrusion of the nanopore; blue dashed line). $\mathrm{V}_{\mathrm{d}}=0 \mathrm{mV}$, whereas $\mathrm{pu}_{\mathrm{u}}$ and $\mathrm{p}_{\mathrm{d}}$, charge density on the pore wall protrusions and ion (and glutamate ${ }^{-}$) concentrations at the upper and lower controlling edges were as in Figure 2.

\subsection{Radial water velocity}

As color coded 2D distributions show the radial water velocity (VWrad; positive velocity movement towards the wall; negative velocity - movement towards the pore center) changed directions at the level of valleys not protrusions, and the inward movement at the level of one valley was followed by an outward movement at the level of next valley. (Figure 8; top panels).

VWrad profiles at $\mathrm{r}=1 \mathrm{~nm}$ and at $\mathrm{r}=2.5 \mathrm{~nm}$ were qualitatively similar, and did not change greatly if the axial position of the object changed (Figs. 8A-D). The object perturbed this pattern, but the effect was limited for the sizes of the object, protrusions and valleys and their spacing. However, if the 
external electric field was reversed, an inward water movement became an outward movement (and vice versa; Figs. $8 \mathrm{D}$ and $8 \mathrm{~F}$ ). If the object was partially charged (on the upper surface only) Vwrad profiles changed little regardless of the external electric field (Figs. 8A and 8D or Figs. 8E-F).
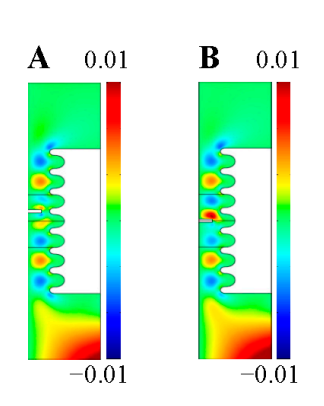

Radial Velocity $(\mathrm{m} / \mathrm{sec})$
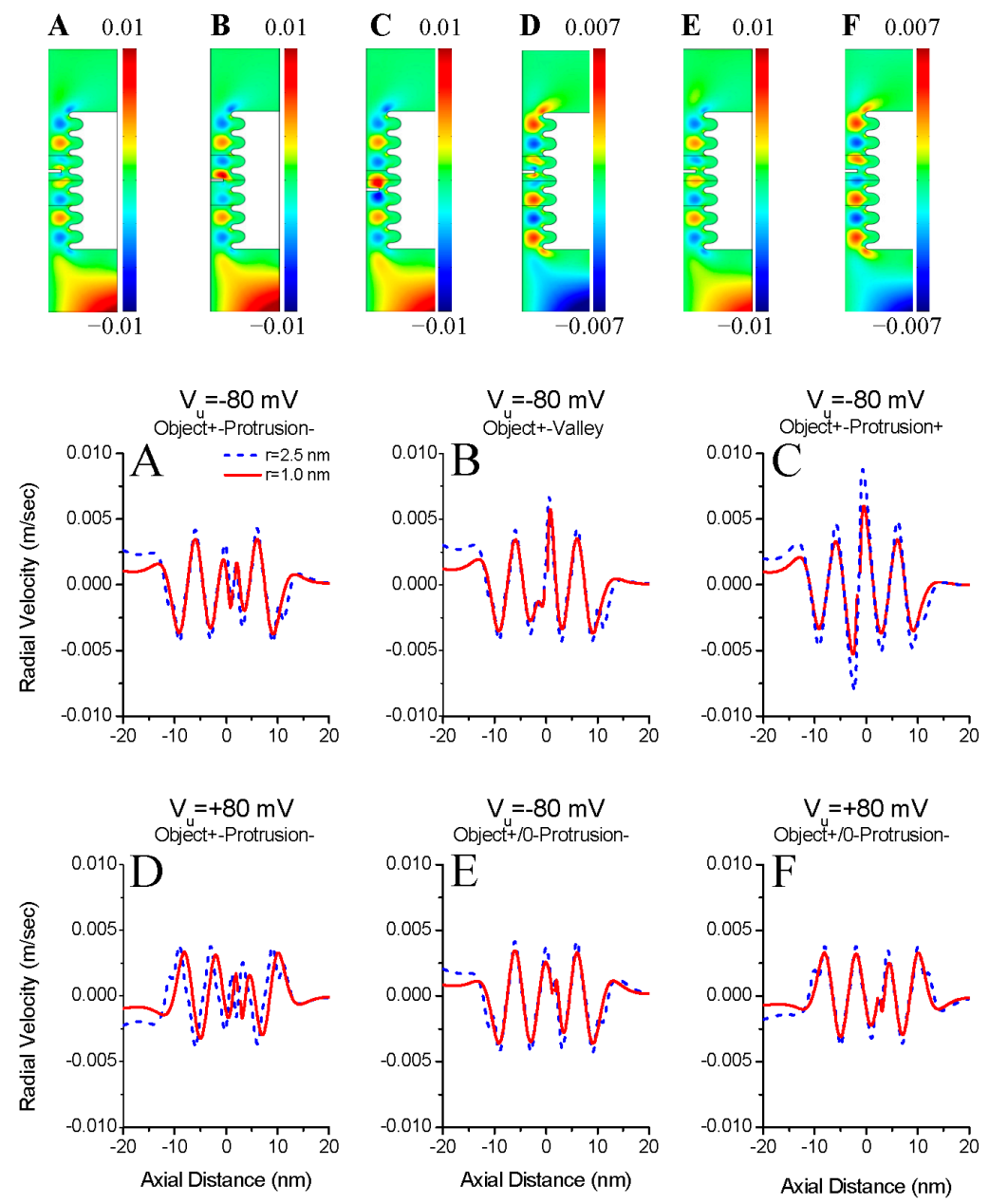

Figure 8. The radial water velocity changes directions at the level of valleys, but if the electric field remains the same (i.e., $\mathrm{V}_{\mathrm{u}}=-80 \mathrm{mV}$ ), the pattern of radial water velocity does not change greatly regardless of whether a positively charged object is positioned opposite a negatively charged protrusion (A), between two oppositely charged protrusions (B), and opposite a positively charged protrusion (C). If $\mathrm{Vu}_{\mathrm{u}}=+80 \mathrm{mV}$, the inward direction of water movement changes to the outward (and vice versa; D). Changing to the partially charged object (the upper surface charged, but not the rest) does not lead to a qualitatively different radial water velocity regardless of the electric field (E-F). The color coded 2D distributions on the top give the radial water velocity. A-F) The radial water velocity at $\mathrm{r}=1 \mathrm{~nm}$ (i.e., at the half disk radius; red solid line) and at $\mathrm{r}=2.5 \mathrm{~nm}$ (i.e., in between the object's end and the maximal protrusion of the nanopore; blue dashed line). $V_{d}$ was $0 \mathrm{mV}$, $\mathrm{p}_{\mathrm{u}}$ and $\mathrm{p}_{\mathrm{d}}$, charge density on the pore wall protrusions and ion (and glutamate ${ }^{-}$) concentrations at the upper and lower controlling edges were as in Figure 2.

\subsection{Hydrodynamic pressure}

Figure 9 top panels depict the color coded 2D distributions of the hydrodynamic pressure for a variety of conditions. Within the nanopore the hydrodynamic pressure was not greatly affected by the object location (panels A-C), by the direction of the external electric field (panels A and D or E and F), or whether the object was uniformly charged or only partially so (panels A and E or D and F). Near the surface of the object the hydrodynamic pressure rose steeply if it was charged (Figs. 9A- 
D), but not if it was not charged (Figs. 9E-F), and it was almost independent on the location of the object vis-à-vis protrusion and its charges. Within the rest of the nanopore it was very low (there was no external pressure; i.e., both $\mathrm{pu}_{\mathrm{u}}$ and $\mathrm{p}_{\mathrm{d}}$ were $0 \mathrm{~Pa}$ ) including in the space between the object and the pore wall (Figures 9A-D). However, if the object was positioned opposite positively charged protrusion, the hydrodynamic pressure rose modestly (Figure 9C).
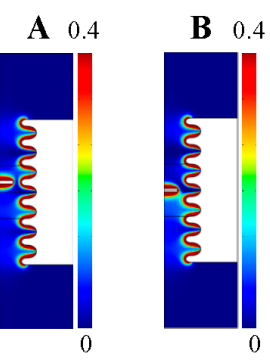

Pressure (MPa)
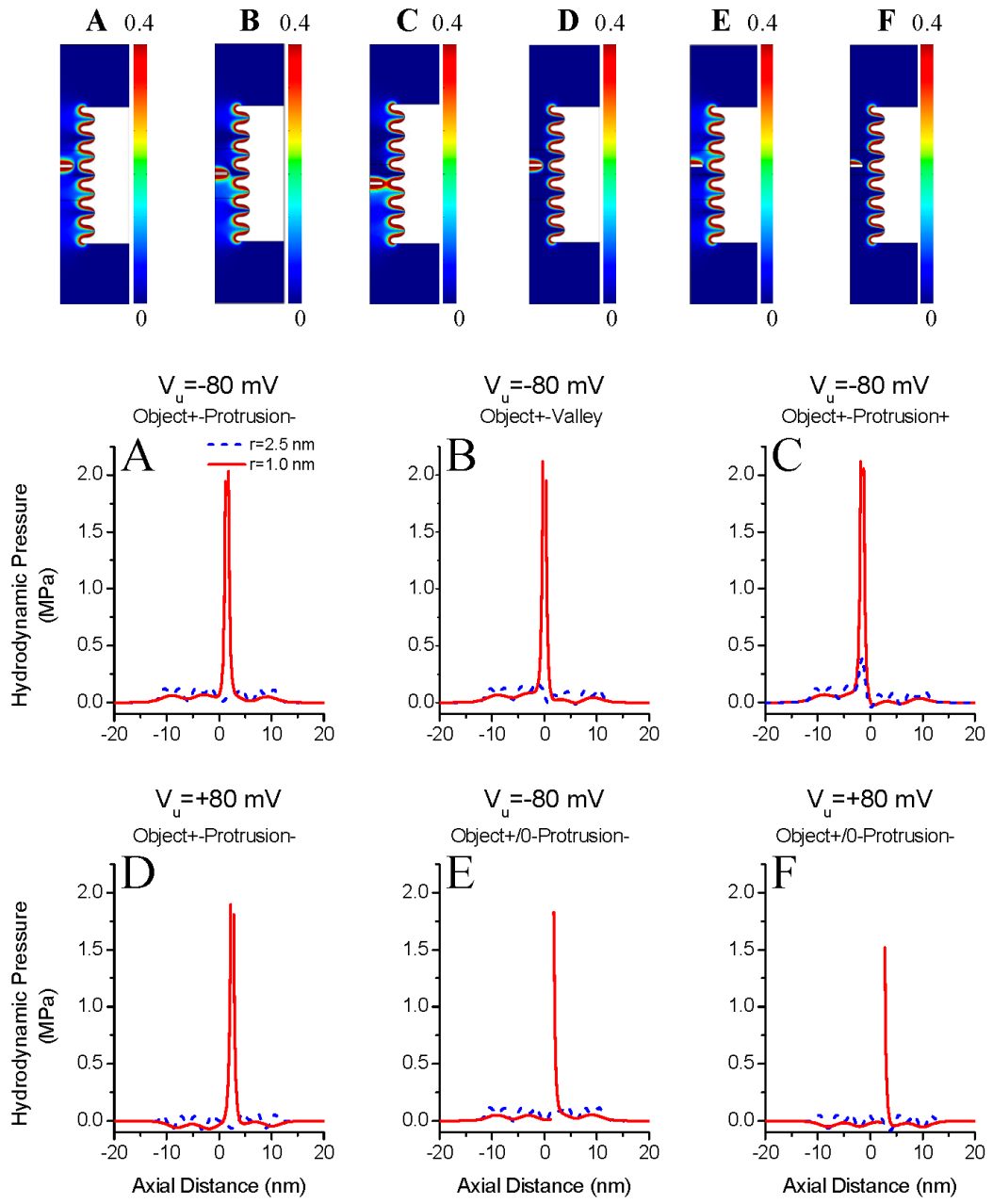

Figure 9. The effect of the electric field and positioning of the charged object towards the pore protrusion and its charging on the hydrodynamic pressure within the nanopore is limited, but the hydrodynamic pressure at the wall strongly depends on the distribution of charge density. A positively charged object is positioned opposite a negatively charged protrusion (A), between two oppositely charged protrusions (B), and opposite a positively charged protrusion (C), and $\mathrm{V}_{\mathrm{u}}=-80 \mathrm{mV}$. The positively charged object positioned opposite a negatively charged protrusion, but $\mathrm{V}_{\mathrm{u}}=+80 \mathrm{mV}$ (D). Partially charged object (upper surface positively charged, but not the rest), with $\mathrm{V}_{\mathrm{u}}=-80 \mathrm{mV}$ (E), and $\mathrm{V}_{\mathrm{u}}=+80 \mathrm{mV}(\mathrm{F})$. The color coded 2D distributions on the top give the hydrodynamic pressure. AD) The hydrodynamic pressure at $\mathrm{r}=1 \mathrm{~nm}$ (i.e., at the half disk radius; red solid line) and at $\mathrm{r}=2.5 \mathrm{~nm}$ (i.e., in between the object's end and the maximal protrusion of the nanopore; blue dashed line).

\section{Discussion}

\subsection{Background}

Advanced fabrication techniques produced nanopores with interesting properties such as elevated pore conductance [6] and ion current rectification [7,8]. The ability to control their transport properties, but also dimensions and over a wide range of scales allows analysis of different types of 
objects, that include proteins [9], DNA [10-12], viruses [13] and nanoparticles [14]. Biomedical and clinical applications include drug delivery [15], and protein sensing and filtration [16]. Nanopore applications include those in engineering as nanofluidic ionic diodes and transistors [17-20] or as ion filters [21].

This study explored the properties of nanopores acting as bio-sensors. As stated in the Introduction size of the particle passing through a nanofluidic pore can be determined, by measuring how much its ion current changes during the transit of particle [1]. However, if long irregular nanopores (i.e., nanopores with axial irregularities) are used nano-size (and meso-size) objects can be distinguished based on their shape. If a non-spherical object transits the nanopore, the extent of the current blockade will depend on its orientation towards the pore axis [22]. It may also rotate, if the diameter of nanopore is equal to (or greater) than its maximal length, and this leads to current fluctuations, which contain information about how non-spherical they are. The sphere-like objects can also rotate, but this does not lead to current fluctuations. This experimentally established method forms a basis of virus characterization [23-25,3]. The presence of axial irregularities appears to be critical for distinguishing the objects by shape, but a full understanding of what forces control the translational and rotational movement of non-spherical objects in axially irregular nanopores is lacking and requires simulations.

The drift-diffusion of $\mathrm{K}^{+}$, glutamate- (or $\mathrm{Glu}^{-}$), $\mathrm{Na}^{+}$and $\mathrm{Cl}^{-}$was simulated within a nanopore using the Poisson-Nernst-Planck equation [4], and was coupled to the transport of water using the Navier-Stokes equations [5]. All simulations were stationary and three-dimensional that were based on axial symmetry. The computational domain consisted of a charged irregularly shaped and comparatively long cylindrical nanopore flanked by two compartments that were separated by the membrane. We used both ions $\left(\mathrm{K}^{+}, \mathrm{Na}^{+}\right.$and $\left.\mathrm{Cl}^{-}\right)$, and molecules (glutamate-) as charge carriers, and although their diffusion constants differ, they do not differ greatly. The concentration (of $\mathrm{K}^{+}$, glutamate-, $\mathrm{Na}^{+}$and $\mathrm{Cl}^{-}$) and potential gradients were present between two compartments, but there was no pressure difference.

In evaluating how the non-spherical object (a disk in this study) will move along the nanopore we calculated three pressures (and forces) acting on the object. First - the Coulomb pressure (force/unit area) due to the fixed charges on the surface of the object, and the electric field, that was partly external (due to the potential difference between two compartments flanking the nanopore) and partly local (induced by the presence of fixed charges on the surface of the object, that were counterbalanced by the screening charges in the solution; 26]. Second - the dielectric pressure induced by the permittivity mismatch between the material of the object and the solution, when placed in a non-uniform electric field [27]. The dielectric pressure is associated with an asymmetric distribution of the electrical stress, which can be determined using the Maxwell stress tensor [27-29]. Third - the hydrodynamic pressure on the surface of the object. The Coulomb and dielectric forces are surface forces, that can be calculated if the electric field on the object surface, the fixed charge density and the permittivity of the object and of the solution are known. The hydrodynamic pressure on the object (also a surface pressure), was calculated simply from the hydrodynamic pressure at the surface. Given that this is a scalar, the direction of the hydrodynamic force due to pressure on the object surface is defined by the surface vector, and on the upper and lower surfaces it has only axial component.

\subsection{Uniformly and non-uniformly charged object}

If the surface of the object was uniformly charged (in this study the charge was always positive and its density was $+64 \mathrm{mC} / \mathrm{m}^{2}$ ), $\mathrm{CP}_{\mathrm{ax}, \mathrm{u}}$ was positive (pointing upwards), but $\mathrm{CPax,d}$ was negative. Both changed quite modestly with radial distance. The axial component of the electric field depended similarly on the radial distance, because the Coulomb pressure is proportional to the electric field and the charge density - a constant in this case (not shown). If however the object was charged only at the upper surface $\mathrm{CP}_{\mathrm{ax}, \mathrm{u}}$, it was modestly positive near the pore center, but became significant and negative near the end of the object. $\mathrm{CP}_{a x, t o t}$ (the sum of $\mathrm{CP}_{\mathrm{ax}, \mathrm{u}}$ and $\mathrm{CP}_{\mathrm{ax}, \mathrm{d}}$ ) on the uniformly charged object can be positive (if $\mathrm{V}_{u}<0$ ), or negative (if $\mathrm{V}_{u}>0$ ). This too changed, if only the upper surface was 


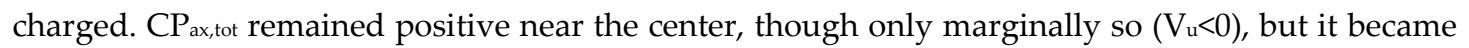
clearly negative near the end of the object. The total Coulomb pressures in the radial direction changed quantitatively, but not qualitatively. $\mathrm{CP}_{\text {rad, }}$ and $\mathrm{CP}_{\text {rad,d }}$ of an uniformly charged object were always positive (i.e., pointing towards the wall of the nanopore) and increased with radial distance. They were similar in value regardless of the external electric field, or whether the object faced a negatively or a positively charged protrusion, or was in-between the two. If the object was charged only at the upper surface $\mathrm{CP}_{\text {rad,u }}$ remained qualitatively the same, but $\mathrm{CP}_{\text {rad, } d}$ was zero. Finally, note that near the end of the object $\mathrm{CP}_{\text {rad,tot }}$ was clearly greater than $\mathrm{CP}_{\text {ax,tot }}$ regardless of whether the object was uniformly charged or not.

The 'dielectric' pressures depended even more on whether the object was uniformly charged. If it was DPax,u and DPax,d were (as expected) significantly smaller and opposite in sign than $\mathrm{CP}_{\mathrm{ax}, \mathrm{u}}$ and

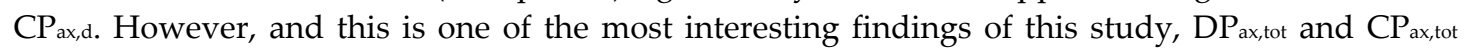
differed far less in value than the corresponding components at each surface, because the total pressure in each case results from the sum of corresponding surface pressures, that were opposite in sign. DPax,tot thus plays an unexpectedly important role in determining how the disk moves axially, and whether it rotates clockwise or counter-clockwise. If the object was positively charged at the upper surface, but not elsewhere (and if $\mathrm{V}_{\mathrm{u}}<0$ ) DPax,u was near zero close to the center, but it was very pronounced and positive near the end of the object. DPax,d was small (but greater than the case in which the object was uniformly charged), and also positive but diminishing near the edge of the object. $\mathrm{DP}_{\mathrm{ax}, \mathrm{tot}}$ was thus positive throughout, and moreover not smaller, but clearly greater than $\mathrm{CP}_{\mathrm{ax}, \text { tot }}$ all along the length of the object. Near the object's end it made a dominant contribution to the combined axial pressure, and to rotation of the object which would be very pronounced. Finally, if the object was uniformly charged DPrad,u and DPrad,d at both sides tend to move the object away from the wall of the nanopore, but were significantly smaller than $\mathrm{CP}_{\text {rad,u }}$ and $\mathrm{CP}_{\text {rad,d }}$ that tend to move it towards the wall. However, if the object was only charged at the upper surface DPrad,u and DPrad,d at both sides were positive especially near the end of the object. DPrad,tot thus now acts in the same

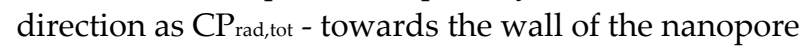

The hydrodynamic pressure $(\mathrm{Pw})$ within the nanopore was quite small (there was no external pressure), but increased steeply to high positive values near both surfaces (upper and lower). Its values were quite similar, if the object was uniformly charged. The hydrodynamic pressure at the upper and lower surface ( $\mathrm{PWu}_{\mathrm{wu}}$ and $\mathrm{PWd}$ ) were negative and positive respectively, being determined by the surface vector. Both decreased in value with radial distance. Being similar in value, Pwtot was very low regardless of how the object was positioned towards protrusions of the nanopore and their charging. The hydrodynamic pressure on the object thus has no effect on the disk movement, but if the pressure (external) existed, and was positive at the upper controlling edges, it would lead to more negative Pwtot and would change the movement of the object (not shown). If the object was nonuniformly charged (i.e., charged at the upper surface, but not elsewhere), Pwu remained as when all surfaces of the object were charged, but Pwd was zero. Pwtot is thus now significant and negative.

Water movement and its velocity was also influenced by presence of the object, its charging and by the irregularity of the nanopore. VWax rose at the level of protrusions (i.e., where effective pore radius diminished) regardless of their charging, and this lead to an alternating pattern of fast/slow axial water velocity that was repeated along the length of the nanopore. VWrad also changed, both its value and its direction, but Vwrad peaks (in either direction) occurred at the level of valleys (not protrusions). The external electric field however influenced direction of the axial water movement, which was largely downwards if $\mathrm{Vu}_{\mathrm{u}}$ was $-80 \mathrm{mV}$, but upwards if $\mathrm{Vu}_{\mathrm{u}}$ was $+80 \mathrm{mV}$.

\section{Conclusions}

In conclusion if the object within an irregular nanopore was uniformly charged, both the total axial Coulomb pressure $\left(\mathrm{CP}_{\text {ax,tot }}\right)$ and total dielectric pressure ( $\mathrm{DP}$ ax,tot) contributed to its axial movement and its rotation. In general, these pressures (and forces) opposed each other, and though the axial dielectric pressure was smaller at each surface $\mathrm{DP}_{\mathrm{ax}, \text { tot }}$ relative to $\mathrm{CP}_{\mathrm{ax}}$,tot was greater than expected and played a surprisingly important role in controlling object movement. The external 
electric field could also be important, and if it was reversed the direction of both $\mathrm{CP}_{\text {ax,tot }}$ and $\mathrm{DPax}$,tot was reversed too. The hydrodynamic pressure within nanopores was very low if there was no external pressure, but near the surface of charged surfaces it rose steeply. Both object surfaces were affected. Pwu was negative whereas $\mathrm{Pwd}_{\mathrm{d}}$ was positive, diminishing near the end of the object. Their total contribution (Pwtot) was however almost zero and did not contribute to either axial movement of the object or its rotation. If the object was charged only at the upper surface the pressures on the object differed greatly. $\mathrm{CP}_{\mathrm{ax}, \mathrm{u}}$ and $\mathrm{CP}_{\mathrm{ax}, \text { tot }}$ were near zero close to the center, but they became negative near its end ( $\mathrm{CP}_{\mathrm{ax}, \mathrm{d}}$ was zero). DPax,tot (which was, but not entirely dominated by $\mathrm{DP}_{\mathrm{ax}, \mathrm{u}}$ ) was positive all along length of the object, increasing greatly near its end. Pwtot, which was identical to $\mathrm{Pwu}(\mathrm{Pwd}$ was zero), was negative and significant, but diminished in value near the end of the object. The axial movement of the object was now determined by Pwtot that dominated along most of the length of the object, and by DPax,tot, which was large at the end and controlled the direction and speed of its rotation. Finally, the water flow pattern was complex.

Acknowledgments: This work is made possible by the grant support to M.I.G from the Natural Sciences and Engineering Research Council of Canada (Grant No 24776).

Author Contributions: Mladen Glavinovic conceived the project; Mohammad Tajparast developed Comsol model; Both authors contributed to writing of the paper.

Conflicts of Interest: The authors declare no conflict of interest.

\section{References}

1. DeBlois, R.W.; Bean, C.P. Counting and sizing of submicron particles by the resistive pulse technique. Rev. Sci. Instr. 1970, 41, 909-916.

2. Heller, I.; Janssens, A.M.; Mnnik, J.; Minot, E.D.; Lemay, S.G.; Dekker, C. Nano Lett. 2008, 8, 591-595.

3. Champion, J.A.; Katare, Y.K.; Mitragotri, S. Particle shape: a new design parameter for micro- and nanoscale drug delivery carriers. J. Controlled Rel. 2007, 121, 3-9.

4. Bockris, J.O.M.; Reddy, A.K.N. 1976. Modern Electrochemistry. Plenum Publ., New York.

5. Temam, R. (2001), Navier-Stokes Equations, Theory and Numerical Analysis, AMS Chelsea Publishing

6. Stein, D.; Kruithof, M.; Dekker, C. Surface-charge-governed ion transport in nanofluidic channels. Phys. Rev. Lett. 2004, 93, 035901-4.

7. Siwy, Z.S. Ion-current rectification in nanopores and nanotubes with broken symmetry. Adv. Funct. Mater. 2006, 16, 735-746.

8. Kovarik, M.L.; Zhou, K.; Jacobson, S.C. Effect of conical nanopore diameter on ion current rectification. J. Phys. Chem. B 2009, 113, 15960-15966.

9. Sexton, L.T.; Horne, L.P.; Sherrill, S.A.; Bishop, G.W.; Baker, L.A.; Martin, C.R. J. Resistive-pulse studies of proteins and protein/antibody complexes using a conical nanotube sensor. Am. Chem. Soc. 2007, 129, 13144-13152.

10. Li, J.; Gershow, M.; Stein, D.; Brandin, E.; Golovchenko, J.A. DNA molecules and configurations in a solidstate nanopore microscope. Nat. Mater. 2003, 2, 611-615.

11. Chen, P.; Gu, J. J.; Brandin, E.; Kim, Y.R.; Wang, Q.; Branton, D. Current characteristic signals of aqueous solution transferring through microfluidic channel under non-continuous DC electric field. Nano Lett. 2004, 4, 2293-2298.

12. Storm, A.J.; Storm, C.; Chen, J.H.; Zandbergen, H.; Joanny, J.F.; Dekker, C. Fast DNA translocation through asolid-state nanopore. Nano Lett. 2005, 5, 1193-1197.

13. DeBlois, R.W.; Wesley, R.K.A. Sizes and concentrations of several type $C$ oncornaviruses and bacteriophage T2 by the resistive-pulse technique. J. Virol. 1977, 23, 227-233.

14. Lee, S.; Zhang, Y.; White, H.S.; Harrell, C.C.; Martin, C.R. Electrophoretic capture and detection of nanoparticles at the opening of a membrane pore using scanning electro-chemical microscopy. Anal. Chem. 2004, 76, 6108-6115.

15. Duan, R.; Xia, F.; Jiang, L. Constructing tunable nanopores and their application in drug delivery. ACS Nano 2013, 7, 8344-8349.

16. Plesa, G.; Kowalczyk, S.W.; Zinsmeester, R.; Grosberg, A.Y.; Rabin, Y.; Dekker, C. 
Fast translocation of proteins through solid state nanopores. Nano Lett. 2013, 13, 658-663. dx.doi.org/10.1021/nl3042678,

17. Gijs, M.A.M. Will fluidic electronics take off? Nat. Nanotechnol. 2007, 2, 268-270.

18. Han, J.H.; Kim, K.; Kim, H.; Chung, T. Ionic circuits cased on polyelectrolyte diodes on a microchip, Angew. Chem. Int. Ed. 2009, 48, 3830-3833.

19. Ali, M.; Mafe, S.; Ramirez, P.; Neumann, R.; Ensinger, W. Logic gates using nanofluidic diodes based on conical nanopores functionalized with polyprotic acid chains. Langmuir 2009, 25, 11993-11997.

20. Tybrandt, K.; Larsson, K.C.; Richter-Dahlfors, A.; Berggren, M. Ion bipolar junction transistor. Proc. Natl. Acad. Sci. U. S. A. 2010, 107, 9929-9932.

21. Vidal, J.; Gracheva, M.E. Leburton, J-P. Electrically tunable solid-state silicon nanopore ion filter. Nanoscale Res. Lett. 2007, 2, 61-68. DOI 10.1007/s11671-006-9031

22. Golibersuch, D.C. Observation of aspherical particle rotation in Poiseuille flow via the resistance pulse technique. Biophys. J. 1973, 13, 265-280.

23. Qiu ,Y.; Hinkle, P.; Yang, C.; Bakker, H.E.; Schiel, M.; Wang, H.; Melnikov, D.; Gracheva, M.; ToimilMolares, M.E.; Imhof, A.; Siwy, Z.S. Pores with longitudinal irregularities distinguish objects by shape. ACS Nano 2015, 9, 4390-4397.

24. Zhou, K.; Li, L.; Tan, Z.; Zlotnick, A.; Jacobson, S.C. Characterization of hepatitis B virus capsids by resistive-pulse sensing. J. Am. Chem. Soc. 2011, 133, 1618-1621.

25. McMullen, A.; de Haan, H.W.; Tang, J.X.; Stein, D. Stiff filamentous virus translocations through solid-state nanopores. Nat. Comm. 2014, 5, 1-10.

26. Cetin, B.; Li, D. Dielectrophoresis in microfluidics technology. Electroph. 2011, 32, 2410-2427.

27. Kang, K.H.; Li, D. Dielectric force and relative motion between two spherical particles in electrophoresis, Langmuir 2006, 22, 1602-1608.

28. Kofod, G. The static actuation of dielectric elastomer actuators: how does pre-stretch improve actuation? J. Phys. D: Appl. Phys. 2008, 41, 1-11.

29. Tajparast, M.; Glavinovic, M.I. Elastic, electrostatic and electrokinetic forces influencing membrane curvature. Biochim. et Biophys. Acta - Biomemb. 2012, 1818, 411-424.

30. Perry, R.H.; Green, D.W. 1999. Perry's Chemical Engineers' Handbook, McGraw Hill .

31. Hille, B. 2001. Ionic Channels of Excitable Membranes, Sinauer Associates Inc, Sunderland. 